\title{
Lifestyle-induced stress drives the natural immune oscillations and COVID-19 infections
}

\author{
Swati Kundu ${ }^{1}$, L Roman Carrasco ${ }^{1}$, R. Manjunatha Kini ${ }^{1,2 *}$
}

${ }^{1}$ Department of Biological Sciences, Faculty of Science, National University of Singapore, 117543, Singapore

${ }^{2}$ Department of Pharmacology, Yong Loo Lin School of Medicine, National University of Singapore, 117600, Singapore

${ }^{*}$ Corresponding author. Email: dbskinim@ nus.edu.sg

\begin{abstract}
Coronavirus and COVID-19 infections continue to wreak havoc across the world. Interestingly, the COVID-19 infections and deaths display a clear seven-day cycles. Mathematical analysis using linear mixed-effects models show that this periodicity is not due to reporting errors. We hypothesize that these COVID-19 cycles are related to natural immune cycles which also oscillate every seven days. These immune cycles are regulated by stress and mediated through the endocrine and the central nervous systems. Our routine activities and lifestyle of more stressful weekdays flanked by less stressful, relaxing weekends define the seven-day immune cycles. The synchronized low immunity levels in the population is responsible for repeated seven-day waves of pathogenic infections such as COVID-19. The new understanding of the role of immune oscillations will help in developing strategies to enhance our immunity through modified lifestyle and better, innovative prophylactic and therapeutic approaches against infectious diseases.
\end{abstract}

\section{INTRODUCTION}

The world is reeling under COVID-19 pandemic. It has infected more than 10 million people and claimed 509,209 lives worldwide as of June 28,2020 [1]. Medical personnel and the government agencies have been working $24 / 7$ to treat millions of patients and limit the infections. Research efforts are focused on developing therapeutic and vaccination strategies to overcome the pandemic. We analyzed epidemiology data of COVID-19 and showed that people from East Asian, Middle- 
east and mid-European countries are less affected compared to Western Europe, US and South America [2]. Thus, we proposed that the number and severity of COVID-19 infections could depend on the variations in structure or tissue-specific expression (alternate splicing and accessibility) of target receptor(s) for SARS-CoV2 and highlighted the importance of infectiongenomics or sankramikogenomics ("Sankramik" in Hindi means infection). Here, we describe the cyclic rhythm in the number of COVID-19 infections and deaths. This periodicity may be related to cyclical variations in our immunity. We propose that the natural oscillations in immune cycles, which are triggered due to our lifestyles of more stressful weekdays and relaxing weekends. These findings will aid in developing better strategies for dealing with COVID-19 and other infectious diseases.

\section{METHODS}

All the COVID-19 infection and death data for the analysis were obtained from Worldometer website [1]. We have been observing the rhythmic pattern in the numbers of COVID-19 infections and deaths for the last 13 weeks. Since it is difficult to obtain statistically reliable data with smaller number or sharp increase or decrease in infections and deaths, we analyzed the March 15 to June 12, 2020 data from the world as a whole and seven-of-the-top 30 infected countries in which the periodicity is distinctly visible. These countries have more than 50,000 infections and 5,000 deaths. The data from Brazil, Germany, Italy, Netherlands, Sweden, UK, USA, and the world were compiled. The time series were detrended using the package pracma in $\mathrm{R}$ to isolate the fluctuations in the series [3]. We used linear mixed-effects models to fit the detrended data to the models to: (i) sine transformation of the day of the week time pi (with Monday being 1 and Sunday 7); (ii) day of the week as a categorical variable; (iii) a binary variable that indicated whether the day was a weekday (coded as 0) or weekend (coded as 1); (iv) a quadratic transformation of the day of the 
week; and (v) a cubic transformation of the day of the week. A random effect for the country was used. The models were compared using the Akaike information criterion (AIC). Model (iii) represents the hypothesis that the observed fluctuations are due to a weekend effect by which reported numbers to drop in that period due to logistical reasons.

\section{RESULTS}

The variations in global number of COVID-19 infections and mortalities show strong periodicity of seven days (Fig 1). We analyzed the data for the seven countries, which demonstrated a similar trend (Fig 1). The amplitude of reported infected cases varies from country to country but the periodicity of one week for each cycle remains constant. At first, we considered the possibility that the observed lower rate of infections near weekends could be due to reporting issues including a lesser number of medical staff taking swabs, the delay in diagnostic tests and the data dissemination. If these were true, we would have observed a sharp increase in infections on Mondays or Tuesdays giving letter 'h-like' curves for each week, and the most supported model by AIC would be that distinguishing weekends and weekdays (Table S1). Instead we observed that the number of COVID-19 infections are peaking on Fridays. Mathematical analyses of the detrended number of cases revealed a repeating curve every seven days and the model with the week of the day as a categorical variable was the most supported model. This model indicates that the peak for infected cases is expected to increase from Monday to Friday peaking on Friday, to later start dropping on Saturday and Sunday (Fig 2a). The model representing the hypothesis of underreporting during weekends was the least supported by AIC (Table S2, Table S3). Similarly, the number of deaths peak mostly on Thursdays and Fridays and bottom near the weekends. As no specialized testing is required to identify and document death, the observed numbers do not reflect significant variations in reporting. Further, the mathematical analysis is repeated with number of 
deaths and the peak is not so pronounced on Fridays (Fig 2b). Thus, the observed seven-day periodicity in the COVID-19 infections and deaths are most likely real. A plausible explanation for these periodicities could be natural fluctuations in our system.

\section{DISCUSSION}

We were interested in understanding the factors that determine these periodic fluctuations. Studies have identified various cycles such as the circadian rhythm (24 hours), menstrual cycle (28 days), lunar cycle (29.53 days), and the seasonal changes (5-6 months) [4]. However, none of these cycles could explain the observed periodicity of COVID-19 infections and deaths.

Since SARS-Cov2 virus is transmitted through air-borne droplets and its transmission success depends on the innate immunity of the individual, we considered natural oscillations of immune cycles. Interestingly, these immune oscillations exhibit seven-day cycle similar to the COVID-19 infections and deaths [5]. This immune cycle is observed as fluctuations in the amounts of CReactive Protein (CRP), the inflammatory marker for the activation of the immune system. Although the amplitude of immune oscillations varies among individuals, the pitch of seven days remains the same. This seven-day fluctuations of CRP levels are used for monitoring and treatment of various cancers including lung, ovarian and melanoma [6] (For details, see the references therein). Weekly pattern in the onset of myocardial infarction and stroke are observed with a peak on Mondays [7,8].

An empirical impact of stress on immune function is well established [9]. Our immune system is regulated by the central nervous system and the endocrine system, which are, in turn, affected by stress [10]. Stress activates the sympathetic-adrenergic-medullary and the hypothalamic-pituitaryadrenal axes that impairs the normal signaling of chemotaxis and adhesion molecules expression 
[11]. During stress, the release of pituitary and adrenal hormones such as the adrenocorticotropic hormone, growth hormone, catecholamines and cortisol, increases the production of proinflammatory cytokines (IL-2, IL-6, interferon) and immune cells (Th1 and Th2) [10,12]. An increase in neutrophils, monocytes, CD2+CD26+, CD2+HLA-DR+ T cells and CD19+ B cells; and a decrease in the CD4+ T cells have been observed [13]. Furthermore, lowering stress shows reversal effects on the immune system. Thus, stress plays a key role on immune system through the endocrine and the central nervous systems.

We hypothesize that the stress cycles are due to our lifestyles of regular weekly activities with different stress situations. Stress levels are high on weekdays; the increased stress levels are due to highly regimented educational or professional challenges as well as strings of back-to-back appointments leading to physical and mental exhaustion. This is compounded by the relatively less sleep times on weekdays. Generally, stress levels are decreased on weekends due to longer sleep times, relaxed scheduling and serenity $[14,15]$. Sleep regulates immune system by maintaining the levels of cortisol and catecholamine, and by enhancing antigen presentation in lymph nodes [16]. As mentioned above, cortisol regulates immune system, its levels are higher on weekdays as compared to the weekends [17]. Therefore, variations in stress levels on weekdays-weekends regulate our seven-day immune cycles and pathogenic infections (Fig 3).

These seven-day immune cycles determine our susceptibility to infections such as the current COVID-19 pandemic. Although an individual could get the pathogen into the body at any time of the week, an opportunistic pathogen, such as SARS-Cov2 virus, will infect the individual and establish the disease only when the immune system is weak to allow the progress. As all our immune cycles are synchronized due to weekday-weekend lifestyle-induced stresses, the number COVID-19 infections follow this seven-day fluctuation trend. Currently, upon COVID-19 
infections, about $9 \%$ patients are dying and the death curve is shifted 12 days (average) from the infection curve (Fig S1). As this number is smaller and mostly driven by comorbidity, the death curves merely follow the infection curves with the same seven-day cycle. The variability in the time from infection to death and relatively smaller death numbers make the pattern slightly less clear.

\section{CONCLUSION}

From the above discussions, we hypothesize that weekly oscillations in our immune system that is most-likely regulated by lifestyle-induced stress determines the seven-day periodicity of COVID19 infections and deaths. We propose that other infectious diseases would also follow the same seven-day fluctuation trend. This new understanding may provide improved strategies to overcome COVID-19 caused infections and fatalities and highlights the importance of managing stress and maximizing immune response to fight infectious diseases.

\section{End notes}

Acknowledgements

This work was funded by the Tier-3 grant from the Ministry of Education, Singapore (MOE 475 2015-T3-1-003).

Declaration

All the authors declare no conflict of interest.

Additional Information

Supplementary Information is available for this paper. 


\section{References}

[1] Worldometer. https://www.worldometers.info/coronavirus/. Accessed 28 June 2020.

[2] Kini, RM, Kundu, S. Infection-Genomics of COVID-19: Are some communities resistant? (Under review).

[3] Borchers, HW, Borchers, MHW. (2019). Package 'pracma'.

[4] Palmer, J. An introduction to biological rhythms. Elsevier; 2012 Dec 2.

[5] Stark, J, Chan, C, George, AJ. Oscillations in the immune system. Immunol Rev. 2007;216(1):213-31.

[6] Coventry, BJ, Ashdown, ML, Quinn, MA, Markovic, SN, Yatomi-Clarke, SL, et al. CRP identifies homeostatic immune oscillations in cancer patients: a potential treatment targeting tool? J Transl Med. 2009;7(1):102.

[7] Manfredini, R, Manfredini, F, Boari, B, Bergami, E, Mari, E, et al. Seasonal and weekly patterns of hospital admissions for nonfatal and fatal myocardial infarction. Am J Emerg Med. 2009;27(9):1097-103.

[8] Willich, SN, Löwel, H, Lewis, M, Hörmann, A, Arntz, HR, et al. Weekly variation of acute myocardial infarction. Increased Monday risk in the working population. Circulation. 1994;90(1):87-93.

[9] Segerstrom, SC, Miller, GE. Psychological stress and the human immune system: a meta-analytic study of 30 years of inquiry. Psychol Bull. 2004;130(4):601.

[10] Glaser, R, Kiecolt-Glaser, JK. Stress-induced immune dysfunction: implications for health. Nat Rev Immunol. 2005;5(3):24351.

[11] Ader, R, Felten, DL, Cohen, N. Psychoneuroimmunology (3 ${ }^{\text {rd }}$ ed.). San Diego, CA: Academic Press. (2001).

[12] Chiappelli, F, Manfrini, E, Franceschi, C, Cossarizza, A, Black, KL. Steroid regulation of cytokines: Relevance for Th1 to Th2 shift? Brain corticosteroid receptors: Studies on the mechanism, function, and neurotoxicity of corticosteroid action. An $N Y$ Acad Sci. 1994;746: 204-215.

[12] Dragoş, D, Tănăsescu, MD. The effect of stress on the defense systems. J Med Life. 2010 Feb 15;3(1):10.

[13] Maes, M, Van Bockstaele, DR, Gastel, A, Song, C, Schotte, C, et al. The effects of psychological stress on leukocyte subset distribution in humans: evidence of immune activation. Neuropsychobiology. 1999;39(1):1-9.

[14] Ragsdale, JM, Beehr, TA, Grebner, S, Han, K. An integrated model of weekday stress and weekend recovery of students. Int J Stress Manag. 201118(2):153.

[15] Ryan, RM, Bernstein, JH, Brown, KW. Weekends, work, and well-being: Psychological need satisfactions and day of the week effects on mood, vitality, and physical symptoms. J Soc Clin Psychol. 2010;29(1):95-122.

[16] Besedovsky, L, Lange, T, Born, J. Sleep and immune function. Pflüg Arch Eur J Phy. 2012;463(1):121-37.

[17] Schlotz, W, Hellhammer, J, Schulz, P, Stone, AA. Perceived work overload and chronic worrying predict weekend--weekday differences in the cortisol awakening response. Psychosom Med. 2004;66(2):207-14. 

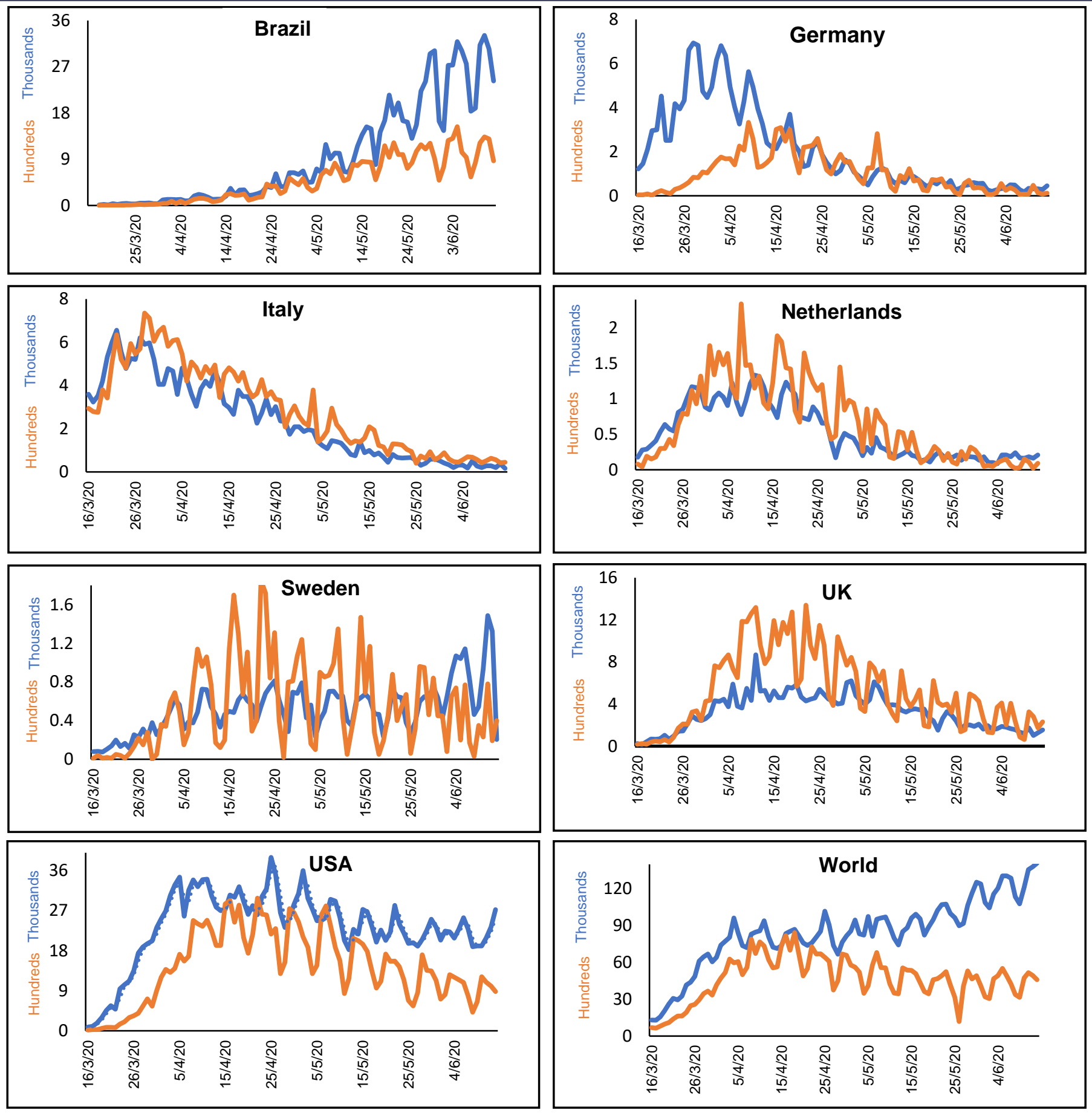

- Number of Infections

Number of Deaths

Fig 1: Periodic oscillation in COVID-19 infections and deaths. The data is from https://www.worldometers.info/coronavirus/. 

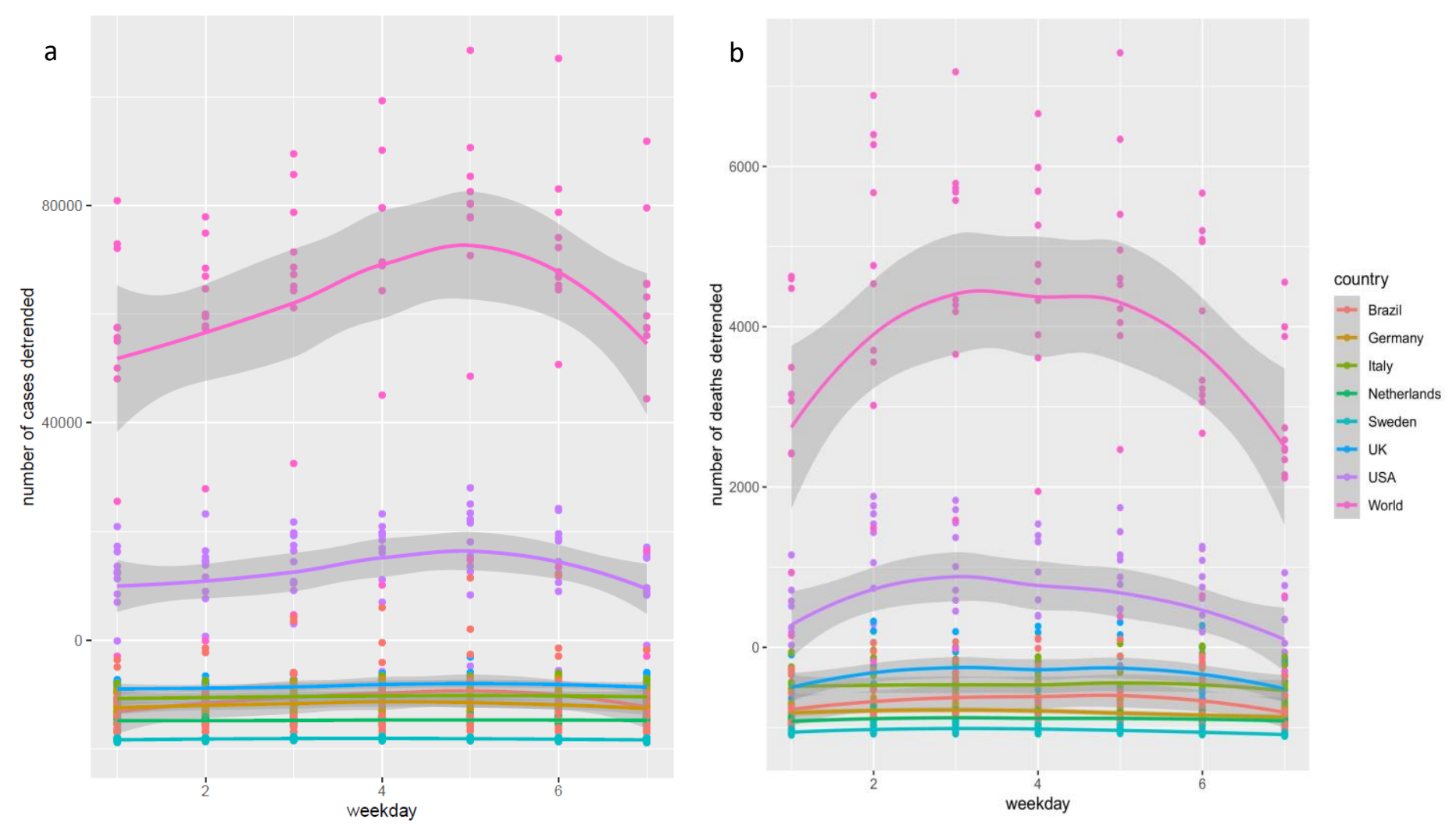

Fig 2: Graphical representation of the detrended number of COVID-19 (a) infections and (b) deaths. Trends are generated using locally estimated scatterplot smoothing.

The data is from https://www.worldometers.info/coronavirus/. 


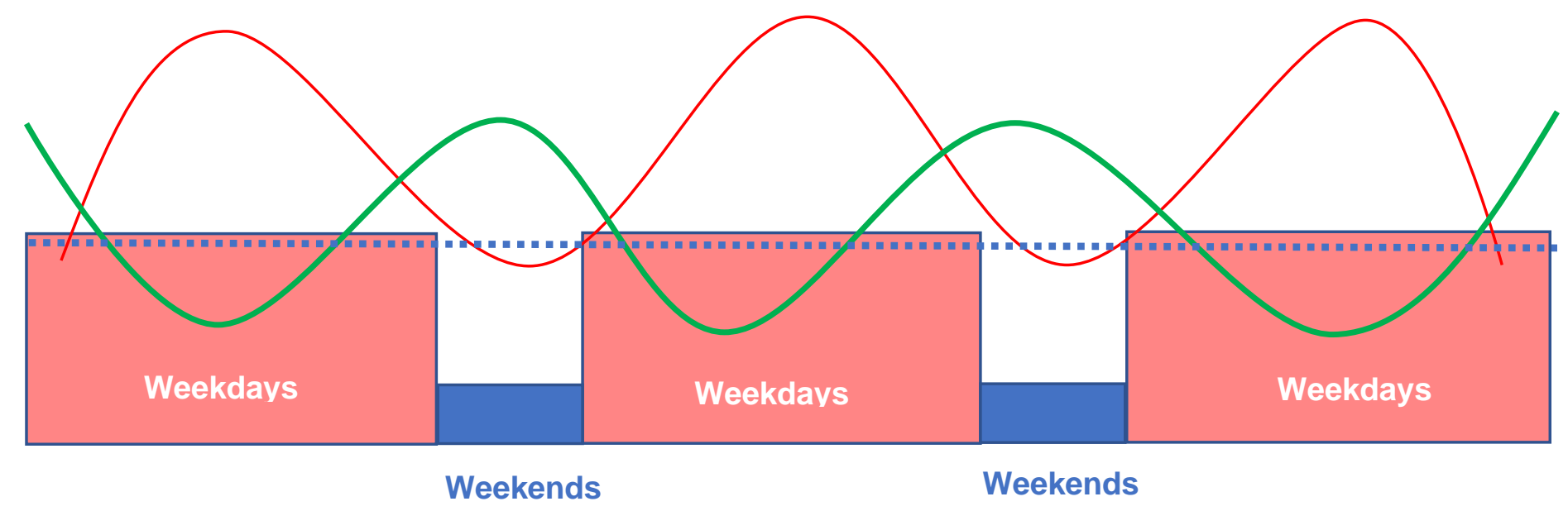

Immune cycles

Fig 3: Lifestyles of high-stress weekdays flanked by relaxing weekends drives Seven-day immune cycles and pathogenic infections.

Stress levels on weekdays are high due to less sleep combined with regimented, back-to-back, intense activities. Stress levels are lower on weekends due to longer sleep times, relaxed scheduling and serenity. Such alternate high and low stress lifestyle drives oscillation in our immunity leading to natural immune cycles (green line). When the immunity drops below the threshold (blue dotted line), opportunistic pathogens take advantage to establish themselves resulting in infections (red line). 


\section{Supplementary Figures \&Tables}
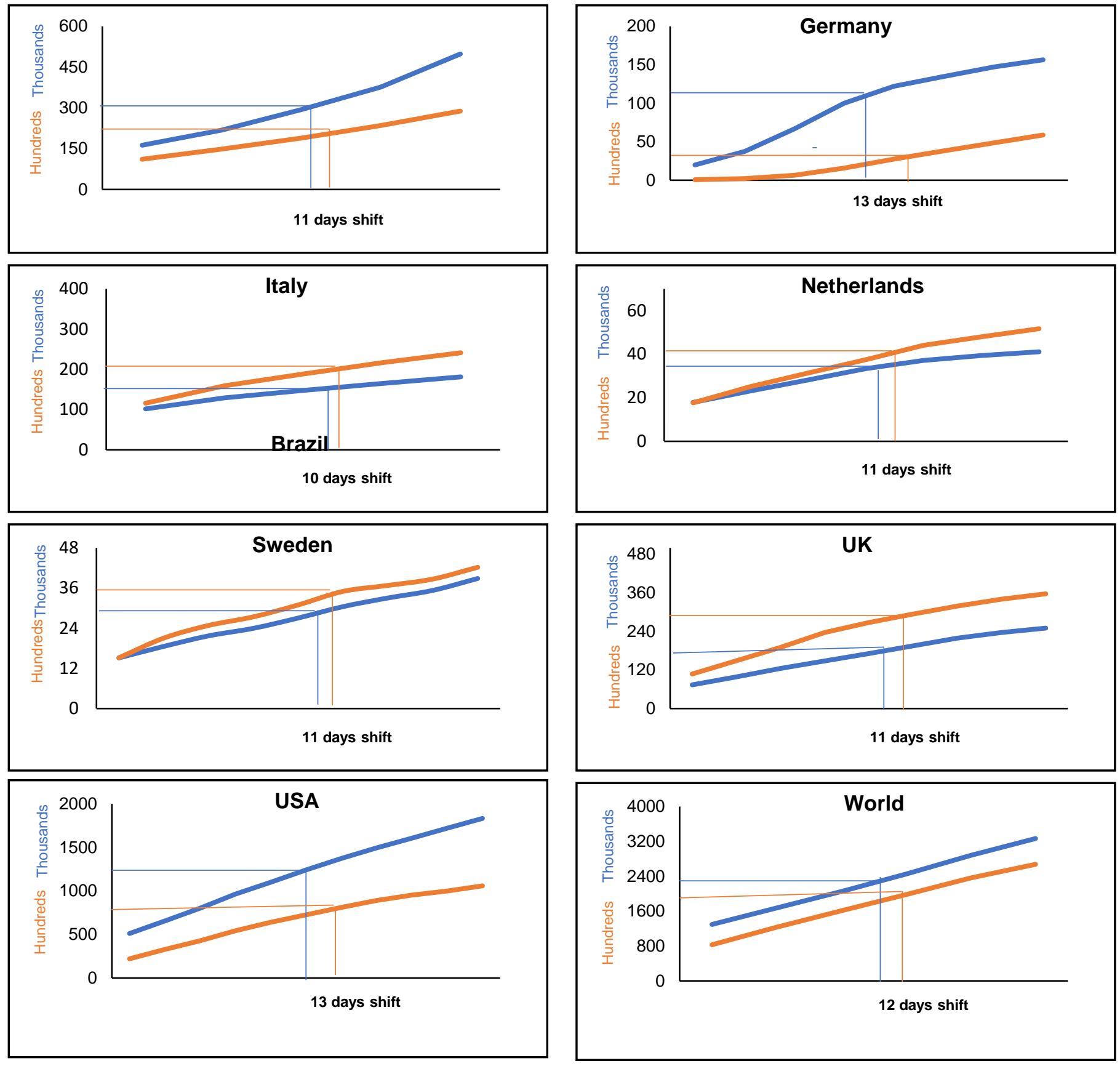

Fig S1: The relationship between COVID-19 infections and deaths.

Only the linear portion of increase in infections and deaths in respective countries and the World are shown. A gap of 10-13 days shown in the number of deaths (50\% shown as orange line) from the number of infections ( $50 \%$ shown as blue line). 
Table S1. Models comparison using AIC.

\begin{tabular}{|c|c|c|c|}
\hline Model & Df & $\begin{array}{l}\text { AIC for number } \\
\text { of cases }\end{array}$ & $\begin{array}{l}\text { AIC for number } \\
\text { of deaths }\end{array}$ \\
\hline model i & 4 & 13154 & 9992 \\
\hline model ii & 9 & 13138 & 9986 \\
\hline model iii & 4 & 13225 & 10053 \\
\hline model iv & 5 & 13205 & 10032 \\
\hline $\operatorname{model} \mathbf{v}$ & 6 & 13194 & 10029 \\
\hline
\end{tabular}

Table S2. Summary of most supported model for number of infections. The intercept corresponds to Monday which is the day with the lowest number of cases.

\begin{tabular}{|l|r|r|r|r|}
\hline & Value & Std.Error & t-value & p-value \\
\hline (Intercept) & -2004 & 9493 & -0.2 & 0.833 \\
\hline Tuesday & 771 & 1420 & 0.5 & 0.588 \\
\hline Wednesday & 2205 & 1420 & 1.6 & 0.121 \\
\hline Thursday & 3257 & 1420 & 2.3 & 0.022 \\
\hline Friday & 4466 & 1420 & 3.1 & 0.002 \\
\hline Saturday & 3112 & 1420 & 2.2 & 0.029 \\
\hline Sunday & 364 & 1390 & 0.3 & 0.794 \\
\hline
\end{tabular}

Table S3. Summary of most supported model for number of deaths. The intercept corresponds to Monday which is the day with the lowest number of cases.

\begin{tabular}{|l|r|r|r|r|}
\hline & Value & \multicolumn{1}{|l|}{ Std.Error } & t-value & p-value \\
\hline (Intercept) & -216 & 563 & -0.384 & 0.701 \\
\hline Tuesday & 338 & 111 & 3.055 & 0.002 \\
\hline Wednesday & 364 & 111 & 3.286 & 0.001 \\
\hline Thursday & 329 & 111 & 2.967 & 0.003 \\
\hline Friday & 346 & 111 & 3.119 & 0.002 \\
\hline Saturday & 223 & 111 & 2.017 & 0.044 \\
\hline Sunday & -62 & 108 & -0.569 & 0.569 \\
\hline
\end{tabular}

